\title{
An Introduction to the Septal Extension Graft
}

\author{
Myung-Hoon Kim, Jeong-Hwan Choi, Min-Su Kim, Seok-Kwun Kim, Keun-Cheol Lee \\ Department of Plastic and Reconstructive Surgery, Dong-A University School of Medicine, Busan, Korea
}

The septal extension graft is a very useful method of controlling nasal lengthening and tip projection, rotation, and shape by fixing a graft to the septum, which leads to a strong supporting structure. Enhancing graft stability is important for better long-term outcomes and minimizing complications or relapse, and even more efficient application of these methods is needed for East Asians who lack enough cartilage to be harvested in addition to possessing a weak cartilage framework. In this paper, the methods for overcoming the drawbacks of the septal extension graft, such as instability, a fixed tip, and insufficiency of cartilage, are presented, and the applications of each method for greater satisfaction with surgical outcomes are also discussed.

Keywords Rhinoplasty / Nasal septum / Transplantation, autologous
Correspondence: Keun-Cheol Lee Department of Plastic and Reconstructive Surgery, Dong-A University School of Medicine, 26 Daesingongwon-ro, Seo-gu, Busan 602-715, Korea Tel: +82-51-240-2968

Fax: +82-51-243-5416 E-mail: pokdungi@dau.ac.kr

This study was supported by research funds from Dong-A University.

No potential conflict of interest relevant to this article was reported.

Received: 13 May 2013• Revised: 20 Jun $2013 \bullet$ Accepted: 30 Jun 2013

pISSN: 2234-6163 • elSSN: 2234-6171 • http://dx.doi.org/10.5999/aps.2014.41.1.29 • Arch Plast Surg 2014;41:29-34

\section{INTRODUCTION}

The septal extension graft, first introduced by Byrd et al. [1] in 1997, achieved fixing a graft on the caudal or dorsal septum between both of the lower lateral cartilages while controlling nasal lengthening and tip projection, rotation, and shape. Compared to the columellar strut in patients with weak midvault or lower lateral cartilage, the septal extension graft has been found to be more favorable for maintaining tip projection through stronger support [1]. A septal extension graft is a very useful tool for East Asian patients, especially those who have a thicker skin and relatively weak lower lateral cartilage, and has become a popular surgical technique in Korea. However, tip drooping or bending of the graft can occur in the case of an insecure fixation, and more efficient design of the graft is needed due to lack of harvestable cartilage, especially in Asians. With these hard and fixed tips, an unnatural appearance is one reason for this technique occasionally not being favored. To overcome the drawbacks of the septal extension graft, various modified techniques have been developed.

\section{THE SEPTAL EXTENSION GRAFT, INTRODUCED BYBYRD}

Byrd et al. [1] found that rhinoplasty for tip control can detach the medial crus from the septum, resulting in weakened tip support, and patients who have weak midvault or lower lateral cartilage are in a high-risk group. The septal extension graft is found to be suitable for these high-risk patients, as tip projection did not decrease in cases with an anchoring intercrural columellar strut to the caudal septum compared to cases without anchoring. Basically, the septal extension graft is formed by fixing the septum through a septal angle, extending to the interdomal space. The distal portion of the graft, forming an appropriate supratip break, is made through a three-point fixation at a lower lateral cartilage along with tip position as needed (Fig. 1). Furthermore, three different procedures for fixing the proximal portion to the septum according to the shape and direction are presented: the paired spreader type, paired batten type, and direct extension type (Fig. 2). The paired spreader type is a graft inserted between the upper lateral cartilage and the dorsal septum via a

Copyright (C) 2014 The Korean Society of Plastic and Reconstructive Surgeons

This is an Open Access article distributed under the terms of the Creative Commons Attribution Non-Commercial License (http://creativecommons.org/

licenses/by-nc/3.0/) which permits unrestricted non-commercial use, distribution, and reproduction in any medium, provided the original work is properly cited.

www.e-aps.org 
spreader graft, extending over the caudal margin. This correction can reinforce a weak midvault and lateralize the upper lateral cartilage, resulting in the widening of the internal valve. However, an oversized graft can collapse the internal valve, and the midvault area can be widened in case of double-layered grafting, and hence dorsal trimming or suturing the posterior portion of the septal angle may minimize the lateralization of the upper lateral cartilage $[1,2]$. Otherwise, grafting the contralateral side as a small batten type would be one of the solutions. Paired battens are fixed diagonally across the dorsal and caudal septum. This procedure does not widen the midvault and has an advantage over the spreader type of tip projection from the supporting vectors [3]. The direct extension type of graft is fixed directly to the anterior septal angle by a horizontal figure-of-eight suture. It cannot be fixed firmly and easily shifts from the midline or does not allow a sustained tip projection and is used only in case of limited available cartilage.

Determining a lateral view intraoperatively is important to avoid excessive extension, which can cause caudal displacement of the

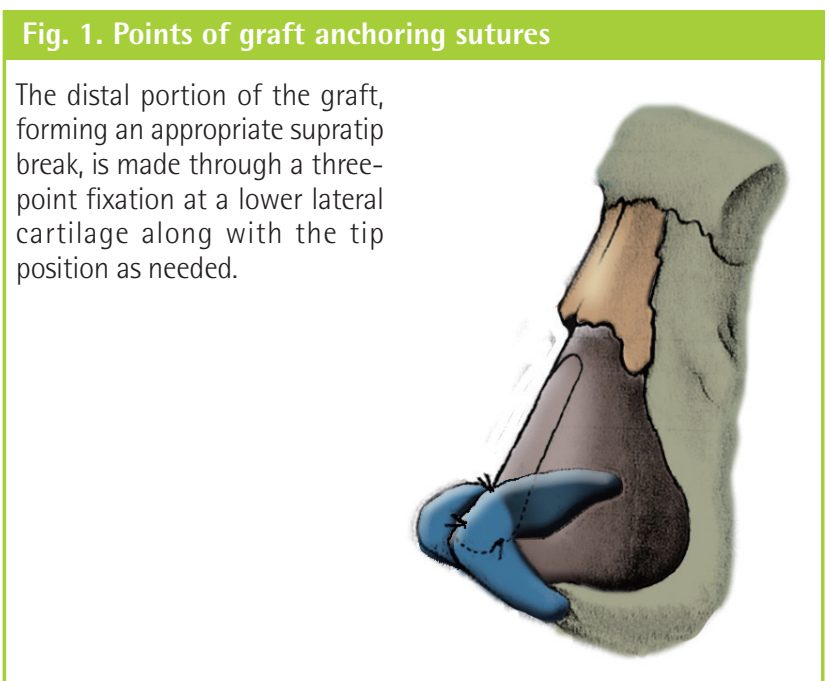

columella, leading to hanging columella. In addition, positioning the graft under the dome and tapering it by a tip-defining suture would prevent the caudal end of the graft from extruding into the interdomal space, which will be seen to broaden eventually [2].

\section{REINFORCEMENT OF STABIILITY}

It is clear that a septal extension graft is a useful tool for tip control; however, sometimes unsatisfactory results can occur unless the graft is fixed securely. A higher stability is needed for the East Asian nose, which, specifically, is thin and has a weak cartilage framework, limitation in cartilage volume available for harvesting, thick skin, and requires greater extension. Various methods that provide strong support have been introduced.

\section{Anchoring suture}

The pivot locking suture

Oh et al. [4] demonstrated a pivot locking suture in which, in addition to the conventional anchoring suture, a figure-of-eight suture is performed on the cephalic and caudal margin where the graft and L-strut meet. This limits the up-and-down movement of the graft, and thereby improves the vertical stability (Fig. 3).

The vertical figure-of-eight suture

Unlike the direct extension procedure of Byrd et al. [1], which presents difficulty in tight fixation, the vertical figure-of-eight suture instead of horizontal figure-of-eight suture yields more stability (Fig. 4). Han et al. [5] measured the shearing, buckling, and tensile force, after using a butt junction type through two simple interrupted sutures, two horizontal figure-of-eight locking sutures, and one vertical figure-of-eight suture. One vertical figure-of-eight suture was the most powerful method. Compared to the butt junction type, methods contacting broadly with the

\section{Fig. 2. Septal extension grafts introduced by Byrd}

(A) The paired spreader type.

(B) The paired spreader type. (C) The direct extension type.

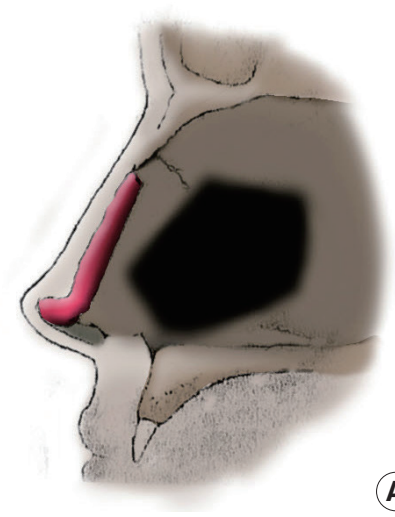

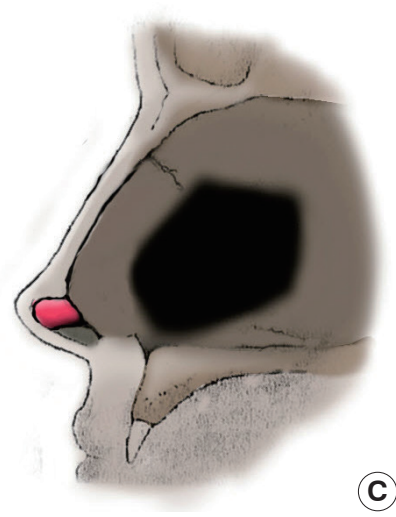




\section{Fig. 3. Diagram of pivot locking suture}

The pivot locking suture limits the up-and-down movement of the graft, and thereby improves the vertical stability.

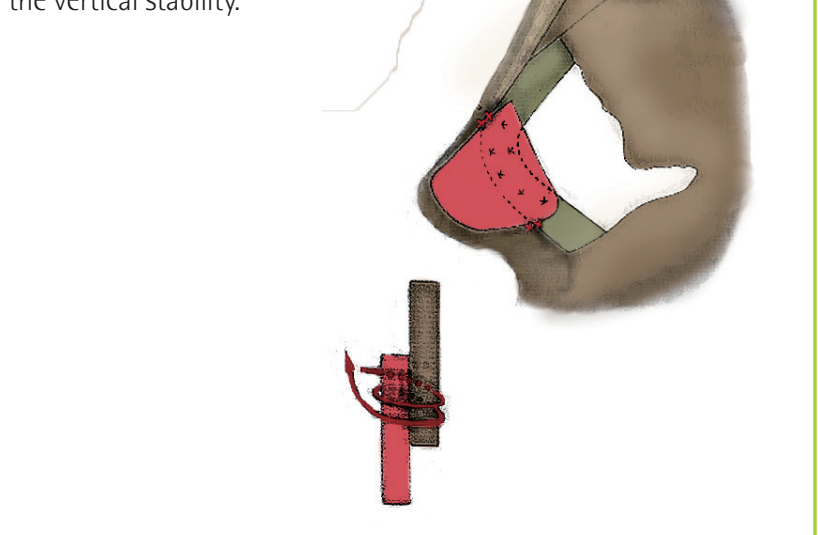

\section{Fig. 4. Diagram of vertical figure-of-eight suture}

A vertical figure-of-eight suture yields more stability than a horizontal figure-of-eight suture.

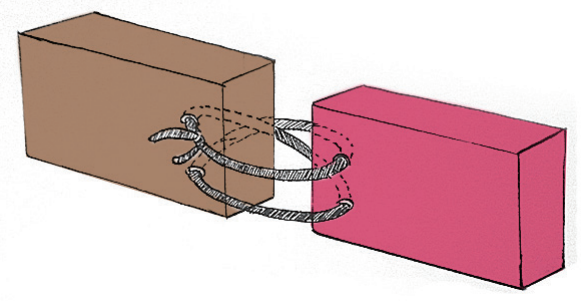

cartilage such as the overlapping type may produce a more powerful fixation, but swallowing of septal mucosa and wastage of cartilage need to be taken into account. Thus, the butt junction type with the vertical figure-of-eight suture may be a good substitution in those who lack available cartilage.

\section{Junction type of the septum and graft}

Byrd et al. [1] also investigated overlapping and direct extension (butt type) grafts. As mentioned earlier, the direct extension is used restrictively because of its difficulty in fixing securely, but overlapping is the most widely accepted method, while other methods of obtaining stability are being introduced. The overlapping type is preferred when the graft is overlapped by more than $4 \mathrm{~mm}$, and in unilateral grafting, the direction of the graft being bent should be considered to minimize asymmetry. Tebbetts [6] described the dovetail junction in fixing the columellar strut to the septum (Fig. 5). By wedging a graft into the 3-5 $\mathrm{mm}$ gap on the caudal septum margin, strong fixation and a low possibility of asymmetry can be gained. However, it is technically difficult and only ideal when the septum is more than 1.5

\section{Fig. 5. Dovetail junction introduced by Tebbetts}

By wedging a graft into the $3-5$ $\mathrm{mm}$ gap on the caudal septum margin, strong fixation and a low possibility of asymmetry can be gained.
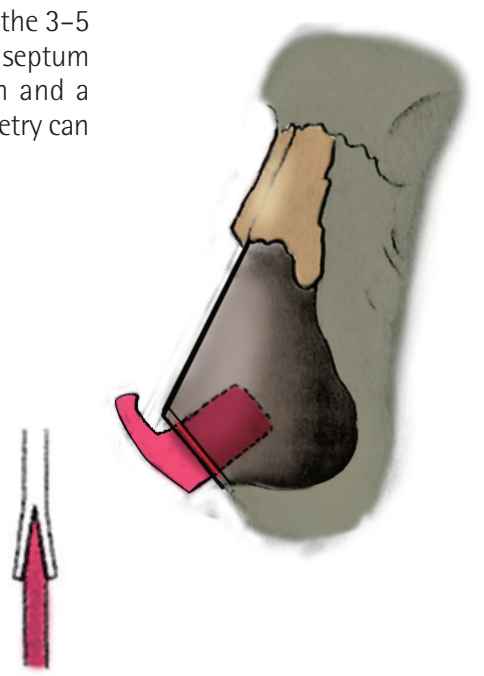

mm thick, and is impractical in East Asians. Lee [7] suggested that an incision on the proximal portion of the graft may induce interdigitation with the caudal septum. Hwang and Hwang [8] measured the contralateral and ipsilateral bending force, and shearing force, after performing a two-point interrupted suture followed by the overlapped graft of $4 \mathrm{~mm}$ and two parallel interdigitated incisions ( $5 \mathrm{~mm}$ each). There were no significant differences between the two groups, and consequently, the overlapping type is more preferred for technical ease.

\section{Direction, design, and additional procedure}

Hubbard [3] described the mechanical advantage according to the direction of the septal extension graft. The spreader type has a mechanical disadvantage in tip projection because of the force vector of soft tissue resistance, but it is advantageous in nasal lengthening or derotation. In contrast, if the graft is located more perpendicularly to the nasal dorsum, it may have a mechanical advantage in tip projection. Moreover, when the tip is deviated to the right or the left, using an inherent bend of the harvested cartilage may be helpful to correct the tip as needed (Figs. 6, 7). Cho [9] suggested a design that decreased the gap between the graft and the anterior nasal spine, leaving some soft tissue to avoid clicking, because drooping can result from long-term pressure to the skin although it is fixed firmly to the septum (Fig. 8). A control columellar strut by Tebbetts $[10]$ is used not only to adjust the shape and angulation of the medial crura but also to broaden the graft width, leading to nasal lengthening by connecting the caudal septum. Furthermore, Kim et al. $[11,12]$ modified this form by enrooting the septal extension graft onto the entire caudal margin of the septum and supporting it through the anterior 


\section{Fig. 6. Mechanical advantage according to the graft direction}

(A) If the graft is located more perpendicularly to the nasal dorsum, it may have a mechanical advantage in tip projection. (B) The spreader type has a mechanical disadvantage in tip projection, but an advantage in nasal lengthening or derotation.
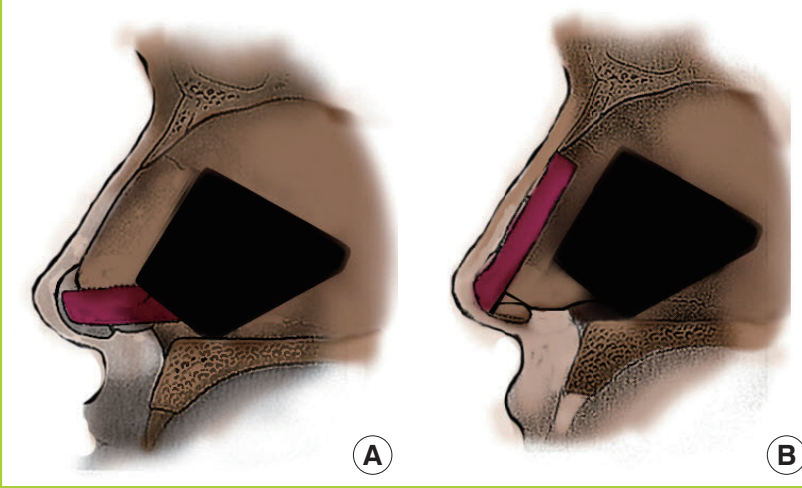

Fig. 7. Correction of tip deviation using an inherent bend

When the tip is deviated to the right or the left, using an inherent bend of the harvested cartilage may be helpful to correct the tip.

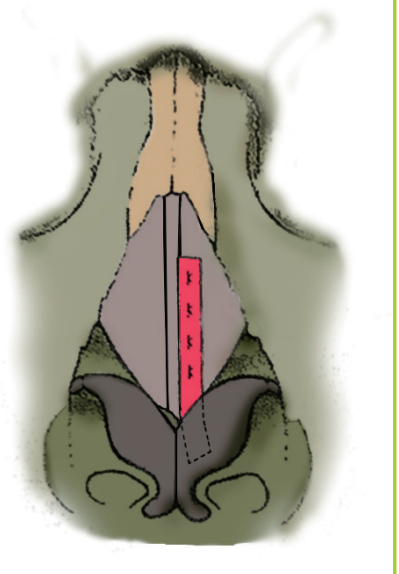

to posterior axis and the caudal to cephalic axis, in turn, effectively correcting even severe columellar retraction. This fixing of the caudal septum lengthways can correct deflection of the caudal septum [13-15]. However, this requires attention to avoid the clicking sound arising from the collision between the graft and the anterior nasal spine following facial expression [16].

Supporting the contralateral side in the other direction is preferred only when a unilateral batten type graft would enhance supporting strength without problems associated with the bilateral and symmetric batten grafts $[17,18]$. In a weak nasal supporting structure, not stable enough with only a septal extension graft, additional procedures such as a derotation graft or tip extension suture should be considered [19].

Relieving pressure on the graft is no less important to stabilizing the tip than providing supporting force to the septal extension graft. Removing contributing factors to tip drooping through detachment of the accessory cartilage linked to the lower lateral

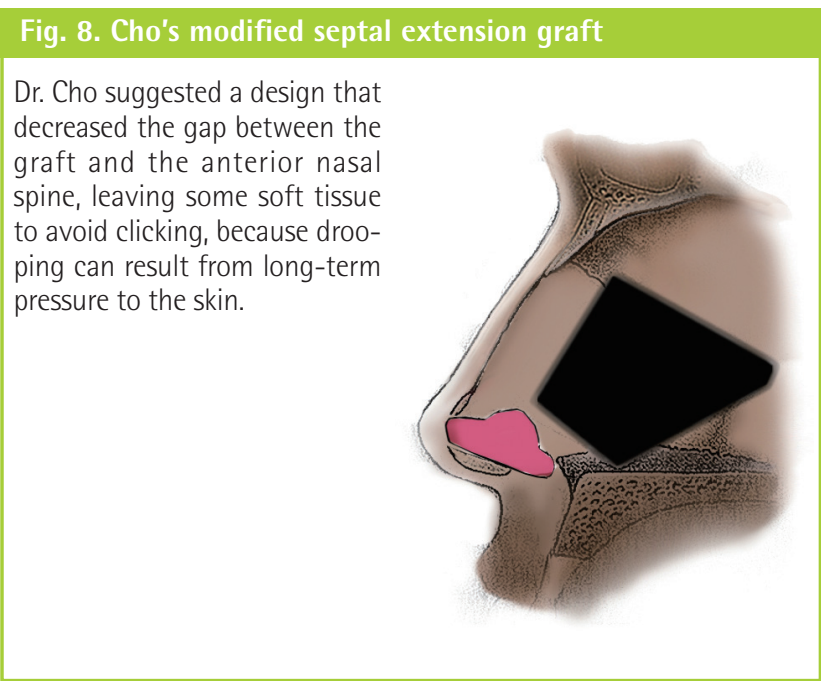

cartilage, dermatocartilaginous ligament, and depressor septi nasi, and also complete release of scar contracture, will minimize tension after extension [20-22]. Thorough bleeding control, adequate drainage, and compressive dressing to reduce postoperative scar formation are essential.

\section{INSUFFICIENT CARTILAGE}

Septal cartilage possessing a strong supporting force is most popularly used for the septal extension graft, but its quantity is often insufficient for harvest. In these cases, a direct extension graft (butt type) can be used, and the surgeon should make every effort to fasten the graft tight, keeping in mind the greater likelihood of deformation than with the overlapping type, as mentioned earlier. Ear cartilage instead of septal cartilage can be applied for reinforcement of the graft such as with the batten type, or for gaining additional tip projection such as an onlay graft. Autogenous costal cartilage can be one of the options, but should be applied selectively due to its excessive thickness and strength and because harvesting it can also leave scars on the chest wall. One of the alternatives, irradiated homologous costal cartilage (IHCC), may relieve cartilage harvesting. Suh et al. $[23,24]$ insisted on a low resorption or infection risk in correction of a contracted nose using IHCC rather than using an autogenous cartilage. Han et al. $[25,26]$ proposed that a septal extension graft using a porous high-density polyethylene sheet (Medpor sheet, Porex Surgical Inc., Newnan, GA, USA) provides a stronger supporting force, especially for severe nasal deformity despite the chance of implant exposure.

\section{HARD AND FIXED TIP}

Since a septal extension graft basically is a fixed type strut to obtain a strong supporting force, problems are not entirely avoid- 


\section{Fig. 9. Kim's modified septal extension graft}

Dr. Kim introduced a design that can maximally preserve the membranous septum.

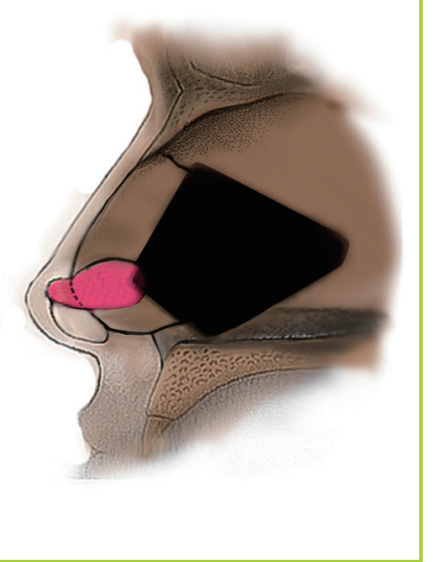

\section{Fig. 10. Diagram of caudal septal advancement}

A rectangular graft design and its advancement in a favorable direction for tip extension from the caudal margin of the septum is suggested.

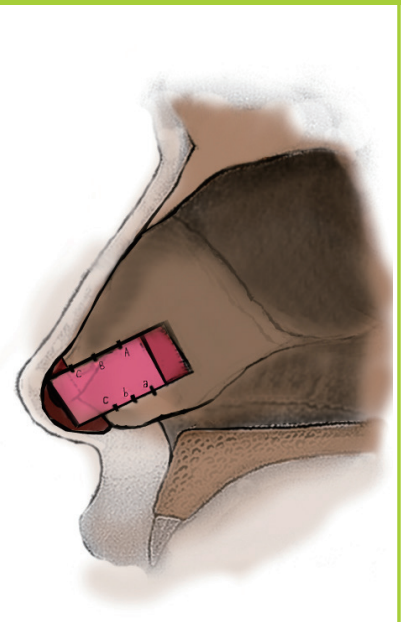

able. With regard to the role of the membranous septum, which is crucial to tip flexibility, a substantial portion of the membranous septum would be attached to the graft to correct the columellar retraction and strengthen support of the caudal septum. Excepting these cases, preservation of membranous septum to the greatest extent possible is helpful for tip flexibility. Kim [19] introduced some designs that can maximize preservation of the membranous septum (Fig. 9).

\section{MODIFIED TECHNIQUES}

Sen and Iscen [27] introduced the caudal septal advancement technique. A rectangular graft design and its advance in a favorable direction as a tip extension from the caudal margin of the septum is suggested. One figure-of-eight suture and two compression sutures each are performed to fix both lateral margins contacting the septum (Fig. 10). This technique does not overlap, so the septum is conserved economically and left intact so

\section{Fig. 11. Diagram of tongue-and-groove technique}

Dr. Guyuron extended the graft by two spreader type struts and a fixed butt type strut in a 'tongue-and-groove' pattern between extended cartilage along the caudal septal margin.

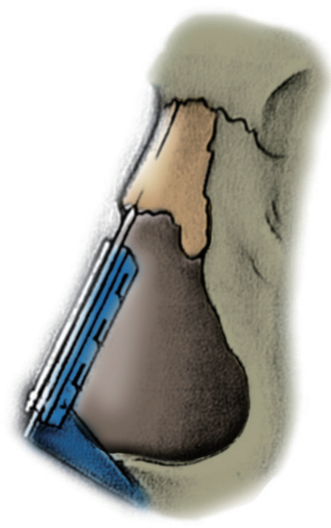

Fig. 12. Kang's modified septal extension graft

Dr. Kang extended the septal extension graft over the bilateral and interdomal space and achieved a desirable tip definition without an additional tip graft. (A) The design for nasal lengthening. (B) The design for tip projection.
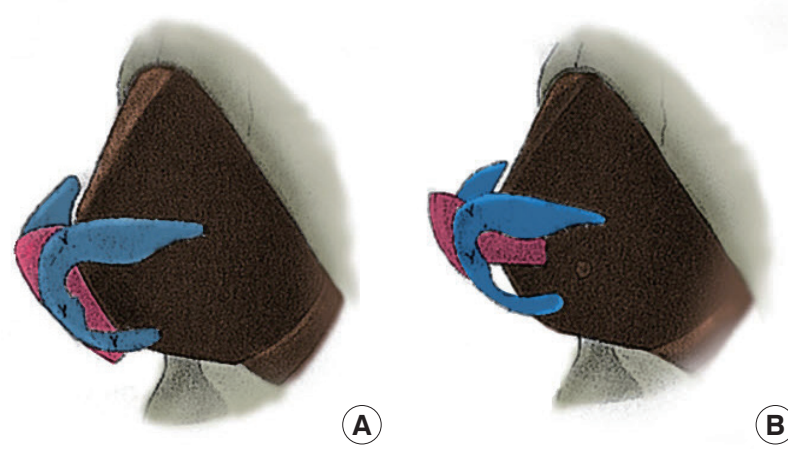

that the lateral and anteroposterior stability is well preserved. However, this method is not adequate in severe septal deviation. Guyuron and Varghai [28] extended the graft by two spreader type and fixed butt type struts in a 'tongue-and-groove' pattern between the extended cartilage along the caudal septal margin (Fig. 11). This has advantages of maintaining symmetry and stability in fixation, but it is frequently difficult to acquire sufficient cartilage.

Kang and Ryu [29] extended the septal extension graft over the bilateral and interdomal space and achieved a desirable tip definition without an additional tip graft (Fig. 12). A pointed tip is rare in a thick-skinned East Asian, and some manipulation to provide a smooth transition is necessary for extension to more than $3 \mathrm{~mm}$.

\section{CONCLUSIONS}

The septal extension graft is an effective method for tip control 
and nasal lengthening with a strong supporting force, but some pitfalls still exist. There are many techniques being introduced to overcome these shortcomings to obtain better outcomes through suitable application of techniques by case. However, the problems have not been fully solved, and thus further efforts and studies are needed.

\section{REFERENCES}

1. Byrd HS, Andochick S, Copit S, et al. Septal extension grafts: a method of controlling tip projection shape. Plast Reconstr Surg 1997;100:999-1010.

2. Ha RY, Byrd HS. Septal extension grafts revisited: 6-year experience in controlling nasal tip projection and shape. Plast Reconstr Surg 2003;112:1929-35.

3. Hubbard TJ. Exploiting the septum for maximal tip control. Ann Plast Surg 2000;44:173-80.

4. Oh SH, Kang NH, Woo JS, et al. Stabilization of unilateral septal extension graft using pivot locking suture. J Korean Soc Aesthetic Plast Surg 2008; 14:156-60.

5. Han K, Jin HS, Choi TH, et al. A biomechanical comparison of vertical figure-of-eight locking suture for septal extension grafts. J Plast Reconstr Aesthet Surg 2010;63:265-9.

6. Tebbetts JB. Primary rhinoplasty: a new approach to the logic and the technicques. St. Louis: Mosby; 1998.

7. Lee HJ. New septal extension graft by "interdigitation" in asian nasal tip plasty. In: 2nd International Congress of Minimal Invasive Plastic Surgery and Dermatology; 2010 Sep 10-12; Seoul: MIPS Committee; 2010. p.239.

8. Hwang SH, Hwang K. Supporting strength of septal extension grafts. J Craniofac Surg 2011;22:2323-6.

9. Cho TY. Tripod projection and lengthening by septal extension graft. In: 7th Asan Rhinoplasty rhinoplasty Symposium; 2009 May 8-10; Seoul: 2009. p49-53.

10. Tebbetts JB. Shaping and positioning the nasal tip without structural disruption: a new, systematic approach. Plast Reconstr Surg 1994;94:61-77.

11. Kim JS, Choi JH, Choi TH, et al. Modified septal extension graft for the correction of nasal tip and columella. J Korean Soc Plast Reconstr Surg 2006;33:681-7.

12. Kim JS, Han KH, Choi TH, et al. Correction of the nasal tip and columella in Koreans by a complete septal extension graft using an extensive harvesting technique. J Plast Reconstr Aesthet Surg 2007;60:163-70.

13. Pham AM, Tollefson TT. Correction of caudal septal deviation: use of a caudal septal extension graft. Ear Nose Throat J 2007;86:142, 4.

14. Seyhan A, Ozaslan U, Sir E, et al. Three-dimensional model- ing of nasal septal deviation. Ann Plast Surg 2008;60:157-61.

15. Jang YJ, Yu MS. Rhinoplasty for the Asian nose. Facial Plast Surg 2010;26:93-101.

16. Daniel RK. Rhinoplasty: an atlas of surgical techniques. 1st ed. New York: Springer-Verlag; 2001.

17. Seyhan A, Ozden S, Ozaslan U, et al. A simplified use of septal extension graft to control nasal tip location. Aesthetic Plast Surg 2007;31:506-11.

18. Huang J, Liu Y. A modified technique of septal extension using a septal cartilage graft for short-nose rhinoplasty in Asians. Aesthetic Plast Surg 2012;36:1028-38.

19. Kim JH. Surgical guides for overcoming disadvantages of septal extension graft in Asians. In: 7th Asan Rhinoplasty Symposium; 2009 May 8-10; Seoul: 2009. p54-7.

20. Pitanguy I, Salgado F, Radwanski HN, et al. The surgical importance of the dermocartilaginous ligament of the nose. Plast Reconstr Surg 1995;95:790-4.

21. Rohrich RJ, Huynh B, Muzaffar AR, et al. Importance of the depressor septi nasi muscle in rhinoplasty: anatomic study and clinical application. Plast Reconstr Surg 2000;105:376-83.

22. O’Keeffe PJ. Dynamic rhinoplasty for the plunging nasal tip: functional unity of the inferior third of the nose. Plast Reconstr Surg 2001;108:1825-7.

23. Suh MK. Contracted short nose correction using irradiated homologous costal cartilage. J Korean Soc Aesthetic Plast Surg 2010;16:117-24.

24. Suh MK, Ahn ES, Kim HR, et al. A 2-year follow-up of irradiated homologous costal cartilage used as a septal extension graft for the correction of contracted nose in Asians. Ann Plast Surg 2013;71:45-9.

25. Han KH, Jeong JW, Park MS, et al. complete septal extension graft using porous high-density polyethylene sheet or septal cartilage in unilateral cleft lip nasal deformities: photogrammetric analysis. J Korean Soc Plast Reconstr Surg 2010;37:400-8.

26. Han K, Jeong JW, Kim JH, et al. Complete septal extension grafts using porous high-density polyethylene sheets for the westernization of the Asian nose. Plast Reconstr Surg 2012;130:106e-15e.

27. Sen C, Iscen D. Caudal septal advancement for nasal tip projection and support in rhinoplasty. Aesthetic Plast Surg 2006;30:135-40.

28. Guyuron B, Varghai A. Lengthening the nose with a tongueand-groove technique. Plast Reconstr Surg 2003;111:1533-9.

29. Kang JG, Ryu J. Nasal tip surgery using a modified septal extension graft by means of extended marginal incision. Plast Reconstr Surg 2009;123:343-52. 